\title{
LE GOUVERNEMENT LULA ET L'ASGENSION POLITIQUE DE LA GRANDE BOURGEOISIE INTÉRIEURE BRÉSILIENNE**
}

Quelles sont les relations entre le premier gouvernement Lula da Silva (20032006) et la bourgeoisie, surtout si l'on adopte une perspective qui rejette l'idée d'une classe capitaliste homogène et qui prend en compte les différentes fractions de la bourgeoisie brésilienne et internationale agissant comme une force sociale aux côtés de l'État brésilien? Critiquant ceux qui soutiennent que le gouvernement Lula est dans la continuité du gouvernement Cardoso, et ceux qui se contentent de dire que le gouvernement Lula est "plus à gauche », cet article se propose d'identifier la classe sur laquelle s'appuie le gouvernement Lula da Silva.

Il soutient que cette base se trouve au sein de la grande bourgeoisie intérieure que Lula a promue, sans rompre, jusque-là, avec l'ancienne hégémonie du grand capital financier national et international. Autrement dit: un gouvernement de base populaire transformé en un gouvernement de cette fraction de la bourgeoise dont les intérêts entrent dans une certaine mesure en conflit avec les intérêts étrangers. De plus, cette analyse montre que les bourgeoisies intérieures des pays dépendants, tels que le Brésil, ne sont pas parfaitement intégrées à la «mondialisation ».

\section{O Governo Lula e a ascensão política da grande burguesia interna brasileira}

O artigo analisa as relações entre o primeiro governo Lula da Silva (2003-200) e a burguesia. $\mathrm{O}$ seu enfoque exclui a idéia de uma classe capitalista homogênea e toma em consideração as distintas frações da burguesia brasileira e internacional que agem como força social junto ao Estado brasileiro. Criticando seja os que sustentam que o Governo Lula é uma continuidade do Governo Cardoso, seja aqueles que imaginam que é suficiente dizer que o Governo Lula está «mais à esquerda », o artigo procura encontrar a base de classe do Governo Lula da Silva.

Sustenta que essa base se encontra na grande burguesia interna que Lula promoveu sem romper todavia com a antiga hegemonia do grande capital financeiro nacional e internacional. Um governo de base popular teria se transformado em um governo da fração burguesa que tem conflitos limitados com os interesses estrangeiros. Essa análise indica além do mais que as burguesias internas dos países dependentes como o Brasil não estão perfeitamente integradas à « globalização ».

\section{The Lula government and the political ascension of the domestic Brazilian bourgeoisie}

The article analyses the relationships between the first Lula da Silva government (2003-2006) and the bourgeoisie, through a perspective which rejects the idea of a homogenous capitalistic class and which bears in mind the various fractions of

* Je remercie mes collègues du projet intégré de recherche Néolibéralisme et rapports de classes au Brésil, du Centro de Estudos Marxistas (Cemarx) de l'Universidade Estadual de Campinas (Unicamp), ainsi que les responsables de ce dossier, pour les critiques apportées à la version originale de ce texte. 
the Brazilian and international bourgeoisie acting as a social force alongside the Brazilian State. Criticising those who maintain that the Lula government is in the continuity of the Cardoso government, and those who confine themselves to saying that the Lula government is "further to the left", this article proposes to identify the class upon which the Lula da Silva government relies. It maintains that this base is to be found in the domestic upper classes, which Lula has promoted without yet breaking from the hegemony of the major national and international financial capital. In other words, a government with a working-class base transformed into a government of that fraction of the bourgeoisie whose interests to some extent enter into conflict with foreign interests. Furthermore, this analysis shows that the domestic upper classes of dependent countries such as Brazil are not perfectly integrated into "globalization".

$\mathrm{L}$ e débat sur les relations entre la bourgeoisie et le premier gouvernement Lula (2003-2006) n'a jusqu'à présent pas dépassé le stade des généralités. Or, il est non seulement nécessaire de débattre de la relation entre ce gouvernement et l'ensemble de la bourgeoisie, c'est-à-dire des intérêts politiques généraux de la classe capitaliste - dimension abordée par les intellectuels critiques - mais aussi des rapports différenciés entre le gouvernement Lula et les différentes fractions de la bourgeoisie brésilienne et internationale, c'est-à-dire les intérêts économiques spécifiques des différents secteurs capitalistes - dimension pour l'instant absente du débat.

Diverses conceptions théoriques dans le domaine de la pensée critique omettent ce type de débat, raison pour laquelle il en est encore à ses balbutiements en ce qui concerne le gouvernement actuel. Il y a ceux qui conçoivent la bourgeoisie comme une classe homogène, c'est-à-dire comme une classe sans la moindre faille significative de fractions aux intérêts économiques spécifiques et d'une présence différenciée sur la scène politique. Il y a aussi la conception développée par Francisco de Oliveira, selon laquelle le processus politique brésilien connaitrait, depuis la fin du modèle "développementaliste », une situation prolongée d'indétermination de classe - les relations entre les classes sociales, leurs fractions et l'activité politique ayant pris fin, rendant le processus politique brésilien « diffus et indéterminé $»^{1}$. Ainsi, l'analyse des classes sociales ne serait plus nécessaire pour comprendre le processus politique.

La thèse du présent article est tout autre. Il ne discute pas directement ces conceptions, mais cherche plutôt à proposer une grille d'analyse, prenant en considération les concepts de bourgeoisie, de fraction bourgeoise et de bloc au pouvoir afin de mieux discerner les relations entre ce gouvernement et les travailleurs.

$\mathrm{Si}$ on considère le problème dans cette perspective, il semble bien que le gouvernement Lula a modifié la relation entre l'État brésilien et la bourgeoisie en améliorant la position de la grande bourgeoisie intérieure des secteurs industriels et de l'agronégoce dans le bloc au pouvoir². Cette ascension n'a pas mis fin à

1 F. de Oliveira, "Política numa era de indeterminação: opacidade e reencantamento », in F. Teixeira da Silva, M. R. Capelari Naxara \& V. C. Camilotti (eds), República, liberalismo, cidadania, Piracicaba (São Paulo), Editora Unimep, 2003 : 191-217.

${ }^{2}$ Le concept de bourgeoisie intérieure a été développé par Nicos Poulantzas pour qualifier la fraction de la bourgeoisie qui occupe une «position intermédiaire » entre la bourgeoisie compradore, 
l'hégémonie du grand capital financier national et international, mais elle s'est produite aux dépens de certaines positions et intérêts de la bourgeoisie financière. Ce changement apparaît principalement dans la politique de commerce extérieur du gouvernement qui a permis une augmentation de l'excédent de la balance commerciale du pays, comparée aux déficits successifs de la balance commerciale brésilienne au long de la décennie 1990. Trois commentaires permettront de mieux comprendre cette approche et ce qu'elle implique.

Tout d'abord, elle se différencie de celles qui n'ont mis en évidence, de façon unilatérale, que les éléments communs entre le gouvernement Lula et les gouvernements de Fernando Henrique Cardoso (FHC), afin de démontrer l'existence d'une continuité politique entre les deux gouvernements ; elle se distingue également des analyses qui différencient le gouvernement Lula des gouvernements FHC en utilisant des qualificatifs génériques et imprécis pour montrer cette différence - le gouvernement Lula serait «à gauche», il serait «plus progressiste» ou «moins néolibéral ", selon les dires, comparé aux gouvernements FHC. En second lieu, il convient d'attirer l'attention sur le fait que la modification qui s'est produite dans le gouvernement Lula représente un déplacement circonscrit à des secteurs du grand capital - déclin du pouvoir du grand capital financier national et international et ascension politique de la grande bourgeoisie intérieure des secteurs industriels et de l'agronégoce; le groupe des petites et moyennes entreprises, qui représente la grande majorité des entreprises du pays, continue d'occuper la même position subordonnée qu'au cours des années 1990, sous les gouvernements FHC. Cela montre la portée limitée, au niveau économique et social, de la modification que représente le gouvernement Lula. Enfin, dans le cadre d'une réflexion sur la mondialisation, parler d'ascension de la grande bourgeoisie intérieure brésilienne signifie que la nouvelle vague d'internationalisation de l'économie capitaliste n'a pas éliminé les intérêts spécifiques de la grande bourgeoisie intérieure de pays semi-périphériques tels que le Brésil. Ce secteur bourgeois continue d'être un acteur important dans les processus politiques nationaux.

\section{L'hégémonie du grand capital financier}

Dans le cadre de la théorie marxiste de l'État, le concept de bloc au pouvoir désigne, comme l'a suggéré Nicos Poulantzas, l'unité contradictoire de la bourgeoisie organisée en tant que classe dominante ${ }^{3}$. Unité de la bourgeoisie, car l'ensemble des capitalistes est favorable au maintien des conditions générales de reproduction du capitalisme; unité contradictoire, car les capitalistes, outre leur unité générale, se positionnent en fonction de la place particulière qu'ils occupent $\mathrm{au}$ sein du processus de production et selon la conjoncture économique et politique d'un pays déterminé, dans des secteurs différenciés qui pourront constituer

qui est une simple extension des intérêts impérialistes dans les pays coloniaux et dépendants, et la bourgeoisie nationale qui, grâce à des mouvements de libération nationale au $\mathrm{xx}^{\mathrm{e}}$ siècle, est parvenue à occuper des positions anti-impérialistes, N. Poulantzas, La crise des dictatures, Paris, Le Seuil, 1976.

3 N. Poulantzas, Pouvoir politique et classes sociales, Paris, Maspero, 1968 ; particulièrement la partie III, «Les traits fondamentaux de l'État capitaliste » : 199-273. 
des fractions de classe ayant des intérêts spécifiques et conflictuels ${ }^{4}$. L'État capitaliste, compte tenu de cette hétérogénéité de la bourgeoisie, joue un rôle actif dans la direction politique prise par le bloc au pouvoir, et il le fait, en général, en hiérarchisant les intérêts des fractions bourgeoises en conflit, de manière à privilégier les intérêts de l'une d'entre elles - qui deviendra la fraction hégémonique.

$\mathrm{Au}$ Brésil, pendant toute la période où le modèle capitaliste néolibéral a été en vigueur, la politique d'État a établi une sorte de hiérarchie parmi des intérêts de la bourgeoisie, façonnant ainsi un bloc au pouvoir néolibéral ${ }^{5}$. Le premier élément du modèle capitaliste néolibéral, consistant à démanteler les droits des travailleurs et les droits sociaux conquis par les travailleurs pendant la période « développementaliste ", répond aux intérêts de toute la bourgeoisie brésilienne et du capital international investi dans le pays. Un tel démantèlement garantit l'unité politique de la bourgeoisie vis-à-vis du modèle. Les grandes entreprises, qui feignent de respecter la législation du travail, en tirent profit quoi qu'il arrive, même si cela se passe indirectement au niveau de leurs fournisseurs et de la pratique du sousemploi, de la déréglementation du marché du travail et de la réduction des coûts que cette déréglementation engendre; la marchandisation de droits et de services tels que la santé, l'éducation et la prévoyance sert, de diverses manières, les intérêts de différents secteurs de la bourgeoisie - on assiste alors à l'essor des affaires d'une fraction bourgeoise que je nomme la nouvelle bourgeoisie de services, directement bénéficiaire du recul de l'État dans le domaine des services de base, tandis que simultanément, on assiste à la réduction des dépenses sociales traditionnelles de l'État, lequel obéit aux pressions du grand capital pour s'approprier le budget public. Le gouvernement Lula poursuit cette politique de déréglementation du marché du travail et de réduction des droits sociaux. De plus, outre la poursuite des réformes promues par FHC, il en prépare de nouvelles - réforme des retraites, réforme universitaire, réforme syndicale et du travail, entre autres.

Ainsi, si le démantèlement des droits des travailleurs et des droits sociaux garantit l'unité politique de la bourgeoisie vis-à-vis du programme néolibéral, les autres éléments du néolibéralisme ont divisé la classe bourgeoise sur le plan de leurs intérêts corporatifs de fraction. C'est en examinant cette division que nous pouvons détecter quels intérêts bourgeois sont prioritairement pris en compte et quels intérêts sont négligés ou rejetés par la politique néolibérale.

Le second élément à considérer dans ce modèle est la politique de privatisation. Cet élément sert directement les intérêts des grands groupes économiques, c'està-dire de l'ensemble du grand capital - national, étranger, industriel et financier. La bourgeoisie moyenne, à cause des règles établies par l'État brésilien pour le processus de privatisation, a été exclue du grand marché des ventes aux enchères d'entreprises publiques. Moins de cent grands groupes économiques se sont appropriés la quasi-totalité des entreprises publiques vendues aux enchères, tout en

\footnotetext{
${ }^{4}$ F. Pereira de Farias, «Sobre a questão das frações de classe dominante », Cadernos Cemarx (Campinas, Unicamp), 1, 2004.

${ }^{5}$ Préalablement à l'analyse du bloc au pouvoir dans le gouvernement Lula, je reprends ici quelques éléments que j'ai développés à ce sujet pour l'ensemble de la période néolibérale ; voir A. Bотто Jr, Política neoliberal e sindicalismo no Brasil, São Paulo, Editora Xamã, 2002, notamment le chapitre I, item 4, «Neoliberalismo e bloco no poder» : 49-76.
} 
bénéficiant de faveurs de toutes sortes - sous-estimation de la valeur des entreprises, possibilité d'utiliser les fameuses moedas podres (titres d'État presque sans valeur de marché), financement subventionné par la Banco nacional de Desenvolvimento econômico e social (BNDES) - principale banque d'État destinée initialement au financement de la production -, informations privilégiées, préférence et aide des autorités gouvernementales, etc. De grandes entreprises industrielles telles que Grupo Votorantim, Gerdau et Vicunha, de grandes banques telles que Itaú, Bradesco, Unibanco et Bozano Simonsen, de grandes entreprises étrangères telles que les entreprises portugaises et espagnoles du secteur de la téléphonie, enfin, le grand capital national, industriel ou financier, et le grand capital étranger, se sont appropriés la sidérurgie, la pétrochimie, l'industrie des fertilisants, les entreprises de téléphonie, la gestion d'autoroutes, de banques publiques, des voies de chemin de fer, etc. Ces entreprises font partie aujourd'hui des entreprises les plus lucratives du capitalisme brésilien. Le gouvernement Lula a hérité de cette privatisation et l'a poursuivie, ainsi que des contrats léonins extrêmement lucratifs pour les nouveaux monopoles privés, sans même envisager de se pencher sur les cas de corruption les plus flagrants concernant la politique de privatisation. De plus, les entreprises se déclarant en difficulté, telle Ferronorte, reçoivent une aide financière privilégiée de l'actuel gouvernement. Les projets de «partenariat public-privé » (PPP), présentés par le gouvernement Lula pour les services publics et l'infrastructure, et le projet loi de privatisation de l'Institut de réassurance du Brésil (IRB), constituent ses plus ambitieuses propositions de privatisation. Ce seront les mêmes grands groupes économiques qui monopoliseront l'accès à l'exploitation des services d'infrastructure avec le privilège d'avoir, comme l'établit le projet des PPP, une rentabilité assurée par la loi - il est prévu de compléter, grâce à des fonds publics, les investissements qui n'atteignent pas la «rentabilité envisagée »; dans le cas de la privatisation de l'IRB, c'est un cadeau spécialement destiné au grand capital financier.

La politique de privatisation est un élément du modèle néolibéral qui touche de façon inégale les différents secteurs de la bourgeoisie. Elle a alimenté, comme nous l'avons indiqué, le patrimoine et les profits du grand capital privé au détriment du moyen capital et elle a nié les intérêts de la bourgeoisie nationale d'État à savoir les hauts fonctionnaires de l'État brésilien qui contrôlaient le réseau des grandes entreprises publiques. La participation de ces entreprises publiques dans le PIB brésilien a fortement chuté au long des deux dernières décennies et l'influence politique de la bourgeoisie nationale d'État a également diminué 6 .

Finalement, en ce qui concerne le troisième élément que nous estimons important dans la politique néolibérale - l'ouverture commerciale et la déréglementation financière - on constate qu'un des secteurs importants du grand capital privé, le secteur industriel lui-même, a vu ses intérêts négligés ou rejetés au profit du grand capital financier national et international.

Afin d'analyser le capital financier et l'actuel modèle d'accumulation, nous ferons référence à François Chesnais, pour qui la forme dominante du capital est

${ }^{6}$ Entre 1989 et 1999, le nombre des entreprises publiques, parmi les 40 plus grandes en activité au Brésil, a chuté de quatorze à seulement sept. E. Dinız \& R. Boschi, Empresários, interesses e mercado, Belo Horizonte, Editora da UFMG, 2004 : 69. 
aujourd'hui celle du capital financier conçu comme «[...] fraction du capital se valorisant en conservant la forme argent ${ }^{7}$. Le grand capital financier au Brésil s'est diversifié en ce qui concerne l'origine du capital, le type d'insertion dans le marché brésilien et le champ d'action. Il y a, avant toute chose, les grandes banques commerciales - nationales et étrangères - qui possèdent un réseau d'agences au Brésil - Bradesco, Itaú, Unibanco, Santander, HSBC, Bank of Boston entre autres. Des données datant de la fin des années 1990 indiquaient que sur un ensemble des 200 banques qui fonctionnaient alors au Brésil, 25 détenaient à elles seules plus de $80 \%$ de l'actif total. Le bilan des profits des banques au cours du premier trimestre 2005 montrait que les cinq plus grandes banques du pays réunissaient $69 \%$ de tous les profits du système bancaire; si on considère les dix plus grandes banques, cette proportion atteint $83 \%$ du total des profits ${ }^{8}$. Au Brésil, les grandes banques et les grands groupes industriels gardent relativement leurs distances entre eux et - particularité brésilienne dans le contexte de l'Amérique latine - les grandes banques nationales représentent un secteur majeur qui d'ailleurs ne comportait pas, jusqu'au milieu des années 1990, d'investisseurs étrangers notoires $^{9}$. Les autres entreprises et institutions qui font partie du capital financier sont les banques étrangères commerciales et d'investissements qui, bien qu'elles n'aient pas d'agences au Brésil, possèdent des investissements à court et à long terme dans le pays, des fonds d'investissements et des fonds de pension nationaux et étrangers. Au Brésil et dans d'autres pays dépendants, ce capital financier fonctionne en grande partie comme capital usurier et prédateur - le capital-argent porteur d'intérêt qui prend de la valeur, à des taux très élevés, sans financer la production capitaliste (possession de titres sur la dette publique, fourniture de prêts au consommateur à des taux qui atteignent $8 \%$ par mois, prêt garanti, c'est-àdire remboursé par prélèvement à la source) à des taux dits «populaires », destiné aux salariés et aux retraités de faible revenu, etc. $)^{10}$.

Pour que le grand capital financier puisse se valoriser rapidement et à des taux élevés, certains aspects de la politique d'État sont fondamentaux dans les conditions actuelles et, principalement, dans les pays dépendants : a) l'intégration du marché financier national aux marchés internationaux, c'est-à-dire la déréglementation financière qui garantit la liberté de conversion des monnaies et de circulation des obligations d'État sur les places boursières; b) un taux de change relativement

7 F. Chesnais, La mondialisation du capital, Paris, Syros, 1997 : 31.

${ }^{8}$ L'enquête a été réalisée par la Banque centrale du Brésil auprès d'un ensemble représentatif de 106 institutions bancaires. Voir «Lucro dos bancos cresce $52 \%$ no $1^{\circ}$ trimestre », Folha de S. Paulo, 4 juin 2005 : B9.

9 Voir A. C. Minella, «Elites financeiras, sistemas financeiros e governo $\mathrm{FHC}$ », in W. J. Rampinelli et N. D. Ouriques (eds), No fio da navalha. Crítica das reformas neoliberais de FHC, São Paulo, Editora Xamã, 1997.

${ }^{10}$ Bien que le capital-argent ne rentre pas dans le procès productif, il fonctionne comme un capital que nous pourrions qualifier d'indirectement productif quand il est prêté au capitalisme actif qui va, lui, le convertir en moyens de production et en force de travail pour générer une plus-value, ce qui n'est pas le cas du capital usurier. Voir les textes récents de François Chesnais, Gérard Duménil, Dominique Lévy, Isaac Johsua et Suzanne Brunhoff qui ont servi de base au Séminaire d'Études Marxistes du premier semestre 2005 à l'École des Hautes Études de Paris; à consulter sur <www.jourdan.ens.fr/levy/sem05.htm>. 
stable et libre qui permette la conversion et la reconversion des monnaies sans crise ni préjudices; c) le paiement de la dette publique externe et interne à un taux directeur élevé, pour assurer une rémunération élevée aux titres publics possédés, en grande majorité, par les entreprises qui ont le plus de liquidités, c'està-dire le capital financier lui-même ${ }^{11}$; d) la liberté pour le capital financier de percevoir le maximum possible pour le capital qu'il cède sous forme de prêt à des capitalistes et à des consommateurs - le spread libre varie, au moment où nous écrivons ce texte, de 60 à $150 \%$ par an, pour un taux directeur de 19,75\% par an; e) un ajustement fiscal qui garantisse le paiement des intérêts des titres de la dette publique - déficit public limité dans les pays européens, excédent budgétaire primaire dans les pays latino-américains. Nous savons que ces cinq éléments ont été conservés, voire approfondis sous le gouvernement Lula.

La déréglementation financière est liée à l'avancée récente de la dénationalisation des économies dépendantes telles que celle du Brésil, ainsi qu'à l'ouverture commerciale promue dans ces pays. D'une part, l'achat et la vente d'actions ou encore l'acquisition d'entreprises publiques et privées est une des options de valorisation du capital financier international, et, d'autre part, les grands groupes industrialofinanciers des pays dominants, ont exigé l'ouverture commerciale de l'Amérique latine pour augmenter leurs exportations vers cette région ${ }^{12}$. Il est notoire que la politique néolibérale a mis fin au protectionnisme des marchés intérieurs latinoaméricains, protectionnisme qui avait été la marque du modèle "développementaliste ». Cette ouverture, outre le fait qu'elle serve les intérêts du capital international, a pour objectif, en acculant la bourgeoisie intérieure à la concurrence des produits importés à plus bas prix, de freiner la hausse du prix de ses produits industriels, en maîtrisant ainsi l'inflation domestique et en contribuant à la stabilité de la monnaie nationale et à la relative stabilité du taux de change. Cette politique a provoqué, pendant le premier gouvernement FHC, une succession de déficits de la balance commerciale, ce qui fut «compensé », au mieux des intérêts du capital financier, par un taux directeur très élevé afin d'attirer le capital financier étranger volatile à la recherche d'une valorisation rapide et forte, et par l'entrée de ce capital-risque à court terme qui compensait donc le déséquilibre de la balance commerciale et des comptes extérieurs. Évidemment, une telle politique risquait ensuite de produire, comme ce fut le cas, une dette publique et un déséquilibre extérieur de plus en plus importants.

L'ouverture commerciale et la déréglementation financière servent, par conséquent, les intérêts du grand capital financier, national et international, au détriment de la grande industrie intérieure. Celle-ci a perdu le marché réservé à ses produits, elle a commencé à payer beaucoup plus cher le capital qu'elle empruntait pour investir et elle a souffert de la diminution de la part de la recette de l'État destinée aux infrastructures et à l'aide à la production.

Pour résumer, tous les aspects de la politique néolibérale - déréglementation du droit du travail et du droit social, privatisation, ouverture commerciale - servent

11 Les bilans des grandes banques privées brésiliennes montrent qu'au cours de ces dernières années, la recette provenant du recouvrement des intérêts des titres de la dette publique représente près de $40 \%$ de la recette totale de ces institutions).

12 F. Chesnais, La mondialisation du capital, op. cit. : 310. 
totalement les intérêts d'une unique fraction de la bourgeoisie: le grand capital financier. Les autres fractions faisant partie du bloc au pouvoir - le moyen capital, la bourgeoisie d'État, le grand capital industriel - sont en conflit, de plus ou moins grande importance, avec un ou plusieurs de ces éléments. Le résultat concret de la concordance objective entre le modèle capitaliste néolibéral et les intérêts financiers est le taux de rentabilité du système financier qui est supérieur à celui du secteur productif. Entre 1994 et 2003, selon les chiffres d'ABM Consulting, les profits des dix plus grandes banques brésiliennes ont tout de même augmenté de $1039 \%{ }^{13}$. Pendant la première année du gouvernement Lula, les banques ont de nouveau obtenu des profits records. Quelques enquêtes menées par Economática et par l'Instituto Brasileiro de Planejamento Tributário (IBPT) ont montré que l'investissement dans les fonds a rapporté, pendant cette période, quatre fois plus que l'investissement dans les secteurs productifs - alors que ce type d'investissement est moins imposé ${ }^{14}$. Au cours du premier trimestre 2005, les profits des banques ont poursuivi leur hausse - ils ont augmenté de $52 \%$ par rapport à la même période en 2004. Cette croissance des profits bancaires semble liée à la dynamique de hausse du taux directeur. En effet, en comparant le premier trimestre 2005 à celui de 2004, on observe qu'entre janvier et mars 2005, lorsque le taux Selic ${ }^{15}$ est passé de $17,75 \%$ à 19,25\%, la recette des banques a été de 19 milliards de réaux; alors que pendant la même période de l'année précédente, lorsque le Selic était à un niveau inférieur et à la baisse, passant de $16,50 \%$ à $16,25 \%$, la recette des banques avec intermédiation financière avait été significativement inférieure -17 milliards de réaux ${ }^{16}$.

Il est donc possible de parler d'une hégémonie du grand capital financier dans le modèle capitaliste néolibéral car, outre les rapports objectifs mis en lumière ci-dessus, il y a également identification politique et idéologique entre les entités nationales et internationales du capital financier et les gouvernements néolibéraux successifs du Brésil. La politique de ces gouvernements, de Fernando Henrique Cardoso à Luiz Inacio Lula da Silva, a été et continue d'être orientée et totalement approuvée par le FMI, la Banque mondiale et la Febraban (Fédération brésilienne des banques) entre autres. Un indicateur significatif de cette situation est la symbiose constatée entre le personnel dirigeant des gouvernements successifs pendant cette période, principalement le personnel du ministère des Finances et de la Banque centrale, et le personnel dirigeant du secteur financier national et international. Commencer sa carrière en tant que directeur de la Banque centrale et la poursuivre comme cadre dans une banque privée, ou bien faire le chemin inverse, est devenu depuis des années un phénomène courant sur la scène politique brésilienne.

\footnotetext{
13 «Lucros dos bancos sobem mais de 1000 \%», Folha de S. Paulo, 21 juin 2004 : B3.

14 «Fundos rendem 4 vezes mais que produção", Folha de S. Paulo, «Caderno Dinheiro », 11 juin 2004 : B1, B3 et B4.

${ }^{15}$ Il s'agit d'un taux de base de la banque centrale qui sert de rémunération aux banques lorsqu'elles souscrivent des titres publics.

16 «Lucro dos bancos cresce 52\% ...», op. cit. : B9.
} 


\section{L'ascension de la grande bourgeoisie intérieure industrielle et de l'agronégoce}

L'hégémonie politique du grand capital financier national et international ne s'est cependant pas imposée sans une certaine résistance et sans connaître certaines variations, tout au long de la période néolibérale. Comme nous l'avons mentionné, la nouveauté du gouvernement Lula en ce domaine est qu'il a promu une opération politique complexe qui a consisté à permettre l'ascension politique de la grande bourgeoisie intérieure industrielle et de l'agronégoce, celle dont les secteurs sont tournés vers le commerce de l'exportation, sans, toutefois, rompre avec l'hégémonie des finances et sans altérer la position subordonnée du moyen capital dans le bloc au pouvoir ${ }^{17}$. Qu'est-ce qui explique ce changement?

Pendant son premier mandat, Fernando Henrique Cardoso a accentué l'ouverture commerciale, enlevant de nouveau toute une série de barrières, douanières et non-douanières, aux importations; il a favorisé la déréglementation de l'entrée et de la sortie de capitaux; il a maintenu un taux de change fort et augmenté le taux directeur et la dette publique. Il a accumulé des déficits croissants de la balance commerciale et a réalisé un ajustement fiscal draconien - qui ne paraît peut-être plus aussi drastique aujourd'hui si on pense au niveau extrêmement élevé de l'excédent budgétaire primaire imposé par le gouvernement Lula au pays. Selon les données de la Banque centrale du Brésil, FHC a obtenu, en pourcentage du PIB national, 0,27\%, 0,08\% et 0,01\% d'excédent budgétaire primaire, respectivement en 1995, 1996 et 1998 ; en 1997, on a enregistré un petit déficit primaire de $0,95 \%$ du PIB. Deux aspects de cette politique ont été particulièrement critiqués par la grande bourgeoisie intérieure: l'ouverture commerciale - le grand capital industriel jugeait qu'elle allait à un rythme trop rapide - et le niveau du taux d'intérêt, thème de réclamations permanentes de la part des industriels. Pendant le premier gouvernement FHC, la Fédération des industries de l'État de São Paulo (Fiesp), secondée par la Confédération nationale de l’industrie (CNI), furent les porte-parole du mécontentement de ce secteur.

Appuyant cette contestation, les grands industriels ont pu compter sur l'aide de la Centrale unique des travailleurs (CUT) et du courant majoritaire du Parti des

17 Pour approfondir ce propos, il faudrait dresser un portrait plus précis de la grande bourgeoisie intérieure et plus spécialement de sa branche exportatrice. Les profits des différents segments de la grande bourgeoisie intérieure sont différemment touchés par le taux de change, selon deux variables au moins : si la fixation du prix de la marchandise de l'entreprise ou du segment est faite en monnaie forte (comme pour les produits agricoles) ou en monnaie nationale (comme pour les produits industriels) et si l'entreprise ou le segment a un degré d'ouverture élevé ou non. Les entreprises et les segments qui présentent un faible degré d'ouverture (ils importent peu de matière première et d'équipements) et dont le prix des marchandises est fixé en réaux, sont les plus touchées par l'appréciation du taux de change; au contraire, les entreprises ou les segments dont les prix des marchandises sont fixés en monnaies fortes et qui présentent un degré d'ouverture élevé, sont les moins touchées par l'appréciation du taux de change. Une grande partie de l'agronégoce occupe une position intermédiaire entre ces deux positions extrêmes. Voir à ce sujet F. Pimentel Puga dans le texte «Câmbio afeta exportadores de forma diferenciada ", in E. Teixeira Torres Filho, F. Pimentel Puga \& F. M. Rocha Ferreira (eds), Visão do desenvolvimento, Rio de Janeiro, Ed. BNDES, 2006 : 65-70. 
travailleurs (PT), principalement de sa section de São Paulo. La CUT, sous la direction d'une nouvelle aristocratie du travail représentée par des travailleurs de l'industrie automobile, du secteur pétrolier et des banques, aspirait, malgré les protestations de la minorité de gauche de la centrale, à la résurgence de l'ancien « développementalisme » que l'on obtiendrait grâce à la réduction du taux directeur et autres mesures d'aide à l'investissement. La proposition d'accords sectoriels tripartites, présentée par la CUT au début des années 1990, était conçue comme un espace privilégié de cette alliance, où des entrepreneurs et des travailleurs de chaque secteur discuteraient, ensemble et avec le gouvernement, des goulets d'étranglement de la production et de l'emploi - financement, impôts, politique d'emploi, etc. Ces accords étaient pensés essentiellement pour le secteur industriel et le seul qui a fonctionné a été l'accord du secteur automobile, annulé postérieurement par le gouvernement FHC. En plus des accords sectoriels, dans de nombreux cas, la Fiesp et la CUT travaillèrent ensemble à l'élaboration de propositions et de projets de politique économique - comme la réforme fiscale, élaborée par la Fiesp et la CUT avec la participation de la faculté d'économie de l'Université de São Paulo (USP), proposition qui avait pour objectif d'« exonérer le capital productif ».

Au moment fort de cette alliance, en juin 1996, la direction de la Fiesp déclara publiquement, dans un texte signé par son président et publié dans la grande presse, qu'elle soutenait la grève nationale contre le chômage organisé par la CUT et par Força Sindical. La Fiesp, pendant les mois de mai et juin de cette année-là, organisa à Brasília, avec la collaboration de la CNI, une manifestation d'industriels de tout le pays contre "le rythme rapide» de l'ouverture commerciale, contre «le rythme lent » des privatisations et contre la politique relative au taux directeur. Le gouvernement FHC, sentant que la pression montait, fit un pas en arrière sans pour autant modifier sa politique générale: il s'appuya sur les normes de l'OMC sauvegarde, droits compensatoires et interdiction de dumping - pour créer des barrières à l'importation de tissus provenant de Chine, de Corée du Sud et de Formose ainsi qu'à l'importation de jouets ${ }^{18}$. Pendant la campagne électorale de 2002, le PT et son candidat Luiz Inacio Lula da Silva se sont efforcés d'obtenir le soutien de la Fiesp, annonçant dans leur discours que leur gouvernement serait celui de la production contre la spéculation. Ils semblaient renouveler les traditionnelles illusions de la gauche brésilienne relative au soi-disant rôle politique de la «bourgeoisie nationale ».

En plus de cette pression politique, un facteur économique doit être pris en compte. Les déficits croissants de la balance commerciale du pays, s'ils servaient les intérêts du capital international, pouvaient, à moyen et à long terme, poser des problèmes au capital financier national et international lui-même. Le déséquilibre des comptes extérieurs, provoqué par le paiement de la dette, par la remise croissante des profits provenant de l'avancée de l'internationalisation de l'économie

\footnotetext{
${ }^{18}$ Les numéros de la revue de la Fiesp publiés entre avril et juillet 1996 couvrent largement ces événements et ils soulignent l'action et les objectifs des industriels - au mois de juin, la publication de la Fiesp a remplacé le titre sobre Notícias («Informations ») par le titre affirmatif Revista da indústria (« Revue de l’industrie »).
} 
et par l'ouverture commerciale elle-même, pouvait compromettre la capacité de paiement de l'État brésilien, voire, si on atteignait un niveau très bas de réserves internationales, mettre fin à la liberté fondamentale du capital financier international d'entrer et de sortir librement du pays. L'économie brésilienne fut proche de cette situation critique lors de la crise du taux de change de 1999, au moment de la transition du premier au second mandat de FHC. Le fantôme de ce que les « développementalistes » de la Commisssion économique pour l'Amérique latine (Cepal) appelaient «étranglement externe » hantait les comptes du Brésil avec l'étranger. La situation exigeait que l'on prenne une autre direction. Le gouvernement FHC, agissant comme l'organisateur de l'hégémonie politique du grand capital financier, sut dépasser les intérêts immédiats de ce secteur. Il démit Gustavo Franco, l'idéologue de l'appréciation du taux de change, de ses fonctions de président de la Banque Centrale, dévalorisa le réal, abandonna la politique de déficit de la balance commerciale et adopta une politique de balance commerciale excédentaire. L'excédent de la balance commerciale et un accord de dernière minute avec le FMI devinrent les atouts dont disposait le second gouvernement FHC (1999-2002) pour redonner confiance au capital financier international dans l'économie brésilienne. Cet embryon de politique d'exportation serait par la suite exploité par le gouvernement Lula ${ }^{19}$.

Il existe donc divers facteurs responsables de la nouvelle politique de commerce international et de l'ascension politique correspondante de la grande bourgeoisie intérieure industrielle et de l'agronégoce. Compte tenu du fatalisme économiciste qui domine les analyses de politique économique au Brésil, il est important de souligner les facteurs politiques qui conduisirent à ce changement - la pression exercée par la grande bourgeoisie industrielle au cours des années 1990, la pression convergente des syndicats et bien sûr, la victoire de la candidature Lula à l'élection présidentielle de 2002. Les facteurs économiques nationaux et internationaux ont également eu leur importance - la menace d'étranglement économique externe qui devint évidente pendant la crise du taux de change de 1999, la croissance du commerce international des matières premières et des ressources naturelles, l'amélioration des cotations de ces produits, le déclin - pendant les années 2000 - du flux de dollars vers les pays dépendants provenant des fonds spéculatifs et des fonds d'investissement des pays dominants, et finalement, la grande dépréciation du taux de change provoquée, involontairement, par la peur du capital international face à la victoire imminente de Lula en 2002. Une fois au pouvoir, Lula décida, de manière radicale, de suivre la voie du changement amorcée par le second gouvernement FHC. Il mit en marche une politique agressive d'exportation, centrée sur l'agronégoce, les ressources naturelles et les produits manufacturés à faible densité technologique, et mit en œuvre des

${ }^{19}$ Les secteurs industriels tournés vers l'exportation ont augmenté leur influence au sein de la Fiesp au cours des années 1990, ce qui permet de comprendre la position actuelle de cette institution vis-à-vis du gouvernement. À ce sujet, voir Á. Bianchi, O ministério dos industriais a Federação das Indústrias do Estado de São Paulo na crise das décadas de 1980 e 1990, thèse de doctorat, IFCH, Unicamp, 2004. 
mesures d'appréciation du taux de change, d'aide au crédit ainsi que d'autres mesures nécessaires au maintien de cette politique.

Ce fut une victoire, bien que partielle, de la grande bourgeoisie intérieure industrielle et de l'agronégoce. Cette fraction bourgeoise se maintint dans une position secondaire dans le bloc au pouvoir, car l'État continua de donner la priorité aux intérêts du capital financier, mais le gouvernement Lula lui offrit une position quand même plus confortable au sein de l'économie nationale. On peut percevoir le résultat de tout cela à travers le comportement de la Fiesp. Cette institution, qui avait critiqué les aspects les plus financiers de la politique économique et de l'ouverture commerciale des années 1990, est aujourd'hui présidée par l'homme de confiance du Planalto (siège du pouvoir exécutif au Brésil), qui fut élu à la Fiesp grâce au soutien du gouvernement fédéral. Le gouvernement Lula a multiplié les canaux institutionnels de consultation d'industriels et d'exportateurs, ce qui contraste beaucoup, selon les témoignages des entrepreneurs euxmêmes, avec ce qui se passait sous le gouvernement FHC. Une recherche sur le milieu des affaires indique, parmi d'autres mécanismes, le lien fort et permanent qui s'est créé entre les associations de l'agronégoce et la diplomatie brésilienne dans la définition de la politique extérieure du pays ${ }^{20}$.

On l'a vu, l'agronégoce, les ressources naturelles et les produits manufacturés à faible densité technologique sont les atouts dont dispose le capitalisme brésilien dans sa course aux dollars. Quelques données supplémentaires doivent être avancées. La participation totale de l'industrie brésilienne par rapport à l'ensemble des exportations du pays a fait un bond dans la décennie 1970, résultat de la politique d'industrialisation de la dictature militaire. De fait, si en 1964 les produits manufacturés représentaient seulement $6,2 \%$ du total des exportations brésiliennes, à peine vingt ans plus tard, au crépuscule de la dictature militaire, cette participation avait fait un bon pour atteindre $56 \%$ du total des exportations! En deux décennies de politique économique industrialisante de la dictature, l'économie brésilienne s'est affirmée comme une grande économie capitaliste périphérique industrialisée. Depuis, la participation des produits manufacturés par rapport à l'ensemble des exportations du pays, malgré de faibles oscillations, a, en termes relatifs, stagné : $54,2 \%$ en $1989,57,3 \%$ en 1994 et $54,7 \%$ en $2002^{21}$. Cela confirme la thèse des critiques de la politique économique des années 1990, selon laquelle le néolibéralisme a provoqué un processus de désindustrialisation au sein des économies d'Amérique latine, ce qui signifie une diminution du poids du produit industriel dans le PIB et que le profil lui-même de l'industrie a changé : déclin des secteurs les plus sophistiqués et ascension des secteurs industriels fondés le plus directement sur les ressources naturelles - minerais, papier et cellulose, produits alimentaires, etc. ${ }^{22}$ Le résultat de cette spécialisation régressive est que

${ }^{20}$ Voir E. Dinız, «Empresários e Governo Lula: percepções e ação política entre 2002 e 2006 », atelier « Empresa, Empresários e Sociedade », Porto Alegre.

${ }^{21}$ J'utilise les données compilées par le géographe A. de OLIVEIRA dans «Os mitos sobre o agronegócio no Brasil », communication au XII Encontro Nacional do MST, São Miguel do Iguaçu, Paraná, janvier 2004.

${ }^{22}$ Alors qu'il fait le bilan de la période 1992-2000, Ricardo Carneiro affirme : «Ce que l'on peut conclure de l'ensemble de ces données, c'est que la structure du commerce extérieur brésilien 
beaucoup de secteurs industriels ont vu leur chaîne de production désorganisée par l'ouverture commerciale et ils sont aujourd'hui déficitaires dans leurs échanges avec l'étranger. Il est vrai que le capitalisme brésilien n'a pas présenté de processus de désindustrialisation aussi important que ceux qui se sont produits au Chili ou en Argentine, mais il n'a pas non plus réussi à se rapprocher des pays asiatiques. Le résultat est que le grand gagnant des relations commerciales entre le Brésil et le reste du monde est encore l'agronégoce. En effet, le solde positif de l'agronégoce brésilien avec l'extérieur a atteint en 2003 le chiffre de 25,8 milliards de dollars, un milliard de dollars de plus que le solde global de la balance commerciale brésilienne cette année-là ; celui-ci avait été célébré car il avait atteint 24,8 milliards de dollars ${ }^{23}$. Autrement dit, sans l'agronégoce, la balance commerciale brésilienne aurait présenté un déficit d'un milliard de dollars en 2003. En 2004, les exportations de l'agronégoce ont totalisé 39 milliards de dollars, ce qui représente une augmentation de $27 \%$ par rapport à 2003 et rend le secteur responsable de $40 \%$ de toutes les ventes du pays vers l'étranger - le complexe du soja vient en tête des exportations, suivi de la viande, du bois, du sucre et de l'alcool, du papier et de la cellulose, du cuir, du café, du coton et des fibres, du tabac et du jus de fruit ${ }^{24}$.

En ce qui concerne la taille des entreprises exportatrices, le grand capital prévaut largement. Selon les données de l'Association brésilienne du commerce extérieur, le Brésil comptait, en février 2005, 19000 entreprises exportatrices. De ce total, seulement 800 entreprises étaient responsables de $85 \%$ du total des exportations du pays. Quant à l'origine du capital, parmi les 40 plus grandes entreprises exportatrices brésiliennes, responsables de $41 \%$ du total des exportations, 22 d'entre elles étaient des entreprises étrangères ${ }^{25}$. Le gouvernement dit qu'il stimule la participation des petites et moyennes entreprises nationales dans le cadre des nouvelles négociations avec la Chine, mais selon les données du Sebrae $^{26}$, dans la branche industrielle, les milliers de micro et petites entreprises exportatrices ne représentent que $2 \%$ des exportations du secteur ${ }^{27}$. La politique de «chasse aux dollars » représente bien une politique qui sert les intérêts du grand capital national et étranger lié à l'agro-industrie, à l'extraction minière et aux produits manufacturés à faible densité technologique. De nouveau, le moyen capital occupe une position subordonnée.

a reflété avec précision les changements qui se sont produits dans la structure productive, ses exportations se concentrant dans des secteurs à faible densité technologique, alors qu'il se passait le contraire au niveau des importations. », R. CARneiro, Desenvolvimento em crise - a economia brasileira no último quarto do século XX, São Paulo, Ed. Unesp, 2002.

${ }^{23}$ A. de Oliveira, op. cit.

${ }^{24}$ «Exportação do agronegócio chega à marca dos US\$ 39 bi », Folha de S. Paulo, 7 janvier $2005:$ B3.

25 «Múltis usam país como base exportadora », Folha de S. Paulo, 17 octobre 2004 : B1.

${ }^{26}$ Le Serviço Brasileiro de Apoio às Micro e Pequenas Empresas (Sebrae) est une institution d'État chargée des micro et petites entreprises

${ }^{27}$ Folha de S. Paulo, «Real valorizado já reduz base exportadora », 27 mai 2005 : B1. 
LES VINGT PLUS GRANDS PROFITS DU PREMIER TRIMESTRE 2005

SELON LES BILANS PUBLIÉS JUSQU'AU 13 MAI

\begin{tabular}{|c|c|c|c|}
\hline Entreprise & Secteur & $\begin{array}{cc} & \text { Variation } \\
& \text { (par rapport } \\
\text { au } & 1^{\text {er }} \text { trimestre 2004) }\end{array}$ & $\begin{array}{l}\text { Profits nets } \\
\text { (millions de réaux) }\end{array}$ \\
\hline Vale do Rio Doce & Sidérurgie/métallurgie & $69 \%$ & 1615 \\
\hline Bradesco & Financier & $98 \%$ & 1205 \\
\hline Banco Itaú & Financier & $30 \%$ & 1141 \\
\hline Usiminas & Sidérurgie/métallurgie & $180 \%$ & 1001 \\
\hline CSN & Sidérurgie/métallurgie & $115 \%$ & 717 \\
\hline Gerdau & Sidérurgie/métallurgie & $81 \%$ & 695 \\
\hline Itausa & Financier & $42 \%$ & 679 \\
\hline Cia. Sid. Tubarão & Sidérurgie/métallurgie & $207 \%$ & 537 \\
\hline Telesp & Télécommunications & $17 \%$ & 490 \\
\hline Unibanco & Financier & $45 \%$ & 401 \\
\hline Banespa & Financier & $1 \%$ & 331 \\
\hline Gerdau Met. & Sidérurgie/métallurgie & $87 \%$ & 312 \\
\hline Telemar & Télécommunications & $21 \%$ & 282 \\
\hline Aracruz & Papier et cellulose & - & 201 \\
\hline Copesul & Chimie & - & 197 \\
\hline Acesita & Sidérurgie/métallurgie & - & 177 \\
\hline Tractebel & Énergie électrique & - & 172 \\
\hline CPFL Energia & Énergie électrique & - & 166 \\
\hline Votorantim & Papier et cellulose & - & 145 \\
\hline Ambev & Aliments/boissons & - & 144 \\
\hline
\end{tabular}

Source: Folha de S. Paulo, «Caderno Dinheiro», éditions des 11, 12 et 13 mai 2005.

Le tableau ci-dessus est un simple instantané trimestriel des profits des grandes entreprises, mais il permet de faire quelques observations. Il montre, en premier lieu, que le gouvernement Lula est un gouvernement très généreux avec les grandes entreprises. En second lieu, la présence majoritaire des grandes entreprises industrielles du secteur exportateur - sidérurgie, papier et cellulose - et des grandes entreprises du secteur financier est significative parmi les entreprises les plus lucratives sous l'actuel gouvernement. Parmi les vingt entreprises citées, quatorze appartiennent à ces deux secteurs. De plus, on observe que ce sont les profits des entreprises sidérurgiques qui ont le plus augmenté au long des douze mois considérés par le tableau, ce qui indique l'importance de la politique d'exportation du gouvernement pour ce secteur de la bourgeoisie. L'autre présence marquante est celle des grandes entreprises privées du secteur des services - énergie électrique et télécommunications. 


\section{Unité et lutte à l'intérieur du bloc au pouvoir}

Pourquoi donc, malgré l'effort du gouvernement en faveur du secteur des exportations et la grande rentabilité que celui-ci présente actuellement, pensons-nous que le grand capital financier national et international continue d'être hégémonique à l'intérieur du bloc au pouvoir sous le gouvernement Lula? C'est parce que ce gouvernement stimule certes la production, mais dans le respect des limites autorisées par les intérêts fondamentaux du grand capital financier.

D'une part, il stimule la production tournée vers l'exportation. Du point de vue des finances, cela n'aurait aucun sens de stimuler la production tournée vers le marché intérieur. Le grand capital financier a besoin de réduire le déséquilibre des comptes extérieurs, sans quoi sa libre circulation et sa forte rémunération pourraient être compromises. L'objectif de cette stimulation de la production doit donc être l'exportation, c'est-à-dire la chasse aux dollars et aux autres devises fortes - ce n'est pas grâce à la consommation populaire interne que l'on obtiendra ces devises. C'est pour cela que l'on stimule particulièrement la production pour l'exportation et non pas la production en général. D'autre part, même dans la politique d'aide à l'exportation, tout doit être fait de façon à ne pas dépasser la mesure de ce qui est de l'intérêt des finances. La course aux dollars, oui ; mais à condition que les dollars obtenus soient utilisés pour payer les intérêts de la dette publique (interne et externe). De cette manière, l'excédent budgétaire primaire et le taux directeur doivent rester élevés même si cela doit limiter la croissance des exportations. De fait, le Brésil d'aujourd'hui manque encore de routes, de silos, de ports, de spécialistes de la surveillance sanitaire et de beaucoup d'autres éléments d'infrastructure et de ressources humaines pour que le capitalisme brésilien puisse s'affirmer comme plateforme d'exportation. Cependant, du point de vue du capital financier, il n'y a aucun intérêt à dévier vers ces infrastructures l'argent qui doit servir à rémunérer les banques. Les points d'étranglement seront, peut-être, dépassés par les " partenariats public-privé », conçus par le gouvernement Lula justement pour surmonter les problèmes d'infrastructure sans menacer la politique d'excédents budgétaires primaires élevés destinés au paiement d'intérêts de la dette publique. Le même raisonnement est applicable à la politique de taux directeur élevé, qui fortifie le profil usurier du capital financier, le détourne du financement de la production et augmente le coût des investissements, limitant ainsi la croissance de l'exportation. C'est la raison pour laquelle, l'excédent budgétaire primaire et les taux directeurs élevés ne sont pas, dans le gouvernement Lula, un avatar financier incrusté dans une politique globalement « développementaliste ». Ils sont, en vérité, cohérents avec cette nouvelle et modeste croissance économique tournée vers l'exportation.

L'augmentation des exportations a été suivie de l'augmentation de l'excédent budgétaire primaire. Celui-ci est passé d'une moyenne de $1 \%$ du PIB pendant le premier mandat de FHC à 3,5\% pendant le second mandat et, maintenant sous le Gouvernement Lula, il est proche de 4,5\%. Si l'on prend en compte la circonstance aggravante que représente un taux directeur également à la hausse, l'affirmation selon laquelle la "chasse aux dollars » est subordonnée aux intérêts des finances prend tout son sens. En résumé, dans la perspective de la fraction hégémonique du bloc au pouvoir, la production doit être stimulée dans la direction (celle du commerce extérieur) et dans la mesure où elle va dans l'intérêt des 
banquiers. Cette limitation montre que la dynamique de croissance économique doit rester modérée et instable. Un tel fait a généré des frictions au sein du gouvernement, c'est-à-dire que l'alliance entre le grand capital financier et la grande bourgeoisie intérieure industrielle et agraire n'exclue pas la confrontation dans la définition de la politique économique. Ces frictions se sont produites à plusieurs reprises tout au long du gouvernement Lula : le conflit entre José Dirceu (Maison civile auprès de la Présidence de la république) et Antonio Palocci (Finances), entre Carlos Lessa (BNDES) et Henrique Meirelles (Banque centrale), entre Dilma Roussef (Maison civile) et, de nouveau, Henrique Meirelles, etc. Dans le cadre théorique de notre analyse, les conflits entre ministères, municipalités, entre un exécutif tout puissant (où le grand capital financier est souverain) et un législatif amoindri (où la représentation politique est plus hétérogène), tous ces conflits, sans écarter d'autres facteurs qui interviennent par ailleurs, doivent être analysés en termes de conflits de fractions bourgeoises au sein du bloc au pouvoir. À l'époque de FHC, il y a déjà eu des conflits de ce type. Mais sous le gouvernement Lula, la dynamique de ces conflits semble indiquer une tendance au renforcement croissant de la grande bourgeoisie industrielle intérieure et de l'agronégoce. Au début de son second mandat, en janvier 2007, Lula a lancé le Plan d'accélération de la croissance - le PAC -, qui semble totalement tourné vers les intérêts de la grande bourgeoisie intérieure, principalement industrielle.

La politique extérieure du gouvernement Lula montre aussi la nouvelle situation du bloc au pouvoir, c'est-à-dire que celle-ci n'est pas déconnectée de la politique intérieure, au contraire de ce que disent ceux qui la considèrent comme la «seule partie saine » de ce gouvernement. Le président Lula dit qu'il lutte pour une nouvelle «géographie commerciale » et c'est là que réside le secret du lien entre sa politique extérieure et sa politique économique. La politique extérieure est à la fois dépendante (vis-à-vis de l'impérialisme) et conquérante (face aux petites et moyennes économies périphériques). D’un côté, on répond aux exigences de l'impérialisme, comme l'envoi de troupes en Haïti, et on réaffirme la position subalterne du capitalisme brésilien dans la division internationale du travail, mais, de l'autre, le gouvernement cherche vraiment à occuper la place qui revient au capitalisme brésilien sur les marchés des produits agricoles, des ressources naturelles et des produits manufacturés à faible densité technologique, même si, pour cela, ce capitalisme brésilien doit s'étendre au détriment des autres bourgeoisies latino-américaines et même si cela génère des tensions commerciales localisées avec certains pays dominants. La lutte contre le protectionnisme agricole de l'Europe et des États-Unis et la détérioration des relations avec l'Argentine illustrent ces affirmations.

L'engagement de l'État brésilien pour construire une alliance d'États périphériques, consacrée par la création du dénommé G-20 lors de la réunion de l'OMC à Cancún en octobre 2003, vise précisément à mettre fin au protectionnisme agricole des pays dominants. Le discours que propose le gouvernement Lula pour légitimer la revendication du G-20, est un discours néolibéral qui plaide pour une « vraie ouverture » des marchés et qui centre la lutte sur le commerce des produits agricoles. Il ne s'agit pas de dénoncer les pays dominants parce qu'ils suivent la maxime hypocrite «faites ce que je dis, pas ce que je fais». Ce que le gouvernement Lula fait consiste à réclamer que le discours néolibéral, c'est-à-dire la fin du 
protectionnisme d'État, soit valable, de fait, pour tous; en procédant ainsi, il renonce à lutter pour des normes qui réguleraient le commerce international et qui favoriseraient les pays dépendants. L'aspect hégémoniste de cette politique ébranle déjà le Mercosur. La grande bourgeoisie intérieure brésilienne, en tant qu'alliée subalterne du grand capital financier, et représentée dans ce cas par l'action du ministère du Développement, de l'Industrie et du Commerce extérieur, aspire à accroître ses parts sur le marché latino-américain et cette aspiration ébranle l'alliance avec le capitalisme argentin au sein du Mercosur.

\section{La grande bourgeoisie intérieure dans la mondialisation*}

Cet article s'est limité au thème du bloc au pouvoir dans cette période néolibérale et, plus particulièrement, à l'examen des relations entre la bourgeoisie et le gouvernement Lula. Nous n'avons pu traiter des relations entre cette bourgeoisie, le gouvernement et les classes travailleuses, ni répondre à la question : "Quel est le processus qui a conduit un parti tel que le PT, créé par le mouvement syndical, à constituer un gouvernement qui représente les intérêts de la grande bourgeoisie intérieure dans ses tensions relatives avec le capital financier international et ses alliés internes? » Nous avons fait référence à cette question à propos de l'alliance entre le syndicalisme ouvrier des usines d'assemblage automobile et la Fédération des industries de l'État de São Paulo, mais n'avons pu l'approfondir. Par ailleurs, quel est le poids des intérêts des travailleurs dans le gouvernement Lula? Pour y répondre, il faudrait examiner les politiques compensatoires développées par celui-ci, ce qui n'était pas possible non plus dans les limites de cet article. On peut néanmoins poser la question de savoir si les politiques d'aide à la production industrielle et à l'agronégoce, tournées principalement vers l'exportation, représentent quand même une amélioration du point de vue des salariés et des agriculteurs brésiliens. Notre analyse suggère que ces politiques reflètent un changement très limité et nous pourrions ajouter qu'elles négligent le développement du marché intérieur et qu'elles créent des difficultés pour la concrétisation de la réforme agraire, dans la mesure où elles entrent en conflit avec les intérêts des travailleurs.

Cependant, il n'est pas correct d'affirmer, comme le font beaucoup d'auteurs, que le gouvernement Lula est dans la continuité pure et simple du gouvernement FHC, car le gouvernement Lula a apporté des modifications, même si elles sont limitées. Il est facile d'indiquer certains aspects généraux du bloc au pouvoir restés inchangés pendant cette période néolibérale - entre le début du premier gouvernement FHC en 1995, et la fin du premier gouvernement Lula en 2006. Pourtant, force est de constater que la hiérarchie du pouvoir bourgeois a adopté, pendant cette période, deux positions extrêmes et opposées en ce qui concerne la politique économique. En haut de l'échelle, le grand capital financier national et international réunit deux attributs privilégiés par la politique d'État - la grande entreprise et l'appartenance au secteur financier. En bas, le moyen capital industriel et agricole tourné vers le marché intérieur, réunit trois attributs rejetés ou négligés par la politique d'État - la petite ou moyenne entreprise, l'appartenance

\footnotetext{
* Sous-titre de la rédaction.
} 
au secteur productif et le recentrage sur le marché intérieur. Entre ces deux positions extrêmes, il y a deux positions intermédiaires. Proche du haut de l'échelle, celle du grand capital industriel et de l'agronégoce, tournés vers l'exportation et, proche du bas, celle des moyennes entreprises exportatrices et du moyen capital bancaire. Ce que le gouvernement Lula a fait a été d'améliorer la position du grand capital industriel et de l'agronégoce tournés vers l'exportation. La possibilité de voir ce secteur parvenir, dans un futur proche, à occuper le haut de l'échelle ne peut être théoriquement écartée - cela signifierait que le gouvernement Lula, pendant son second mandat, qui ira jusqu'en 2010, mette fin à l'hégémonie financière au bénéfice de la grande bourgeoisie intérieure brésilienne.

Ce qu'on appelle la mondialisation n'a pas supprimé les bourgeoisies intérieures des pays périphériques. Cette nouvelle vague d'internationalisation de l'économie capitaliste n'a pas la capacité de rendre homogène l'espace économique mondial, contrairement à ce que suggèrent beaucoup d'auteurs qui travaillent sur ce thème. Dans le cas du Brésil, pays où le capitalisme est le plus fort d'Amérique latine, dans un premier temps, sa bourgeoisie intérieure a résisté à la volonté de l'État brésilien d'encourager l'ouverture commerciale et la déréglementation financière. De fait, la résistance ou réticence de la grande bourgeoisie intérieure pendant les années 1970 et 1980 a été un des éléments responsables du fait que le Brésil a adhéré tardivement aux réformes proclamées par le Consensus de Washington l'autre élément a été la lutte populaire, représentée par la création au début des années 1980, du Parti des travailleurs, de la Centrale unique des travailleurs et du Mouvement des travailleurs ruraux sans terre (MST). Alors que le Chili et l'Argentine ont commencé leurs réformes néolibérales dès les années 1970 et le Mexique dans les années 1980, le Brésil n'a pris cette direction qu'au cours des années 1990 et, dans de nombreux secteurs de l'économie et de la société, les réformes au Brésil furent plus superficielles que celles des pays voisins. Aujourd'hui, après à peine une décennie d'expérience néolibérale, la grande bourgeoisie intérieure revient sur la scène politique pour tenter d'obtenir une réorientation de la politique de développement. Le gouvernement Lula représente, fondamentalement, ce retour. La bourgeoisie brésilienne n'est pas sortie indemne des réformes. Le poids de l'industrie, du capital national et du capital d'État a diminué ; la production pour l'exportation, l'agronégoce, une nouvelle bourgeoisie de services (éducation, santé, prévoyance) ont pris de l'importance. Néanmoins, un puissant secteur de la bourgeoisie continue d'exister qui, bien qu'il n'ait pas d'intérêts ni de pratiques anti-impérialistes, possède des intérêts conflictuels avec le capital financier international et avec ses alliés internes préférentiels. 